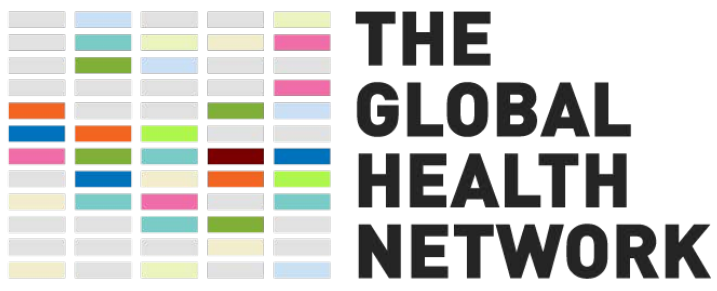

Enabling research by sharing knowledge
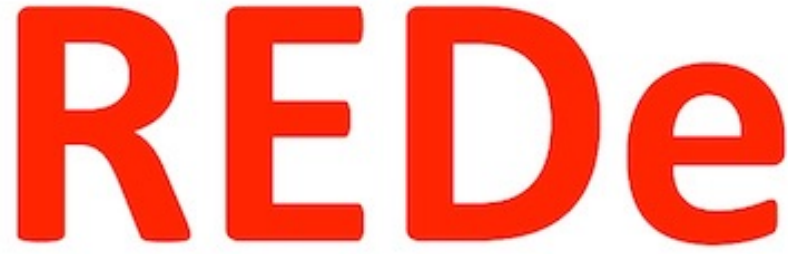

Research Capacity Network

\title{
Establish a Research Support Room in your institution / healthcare facility
}

Authors: Licia Mota, Cleandro Pires de Albuquerque, Guilherme Dias Malvão, Fernando Araújo Rodrigues de Oliveira, Dayde Lane Mendonça da Silva, Elza Noronha.

Design: Mercedes Rumi, Nicole Feune de Colombi, Steffani Herring-Hall

Date of publication: 07-02-2022

Abstract: Step by step guide to create a Research Support Room, an enduring service to offer support for healthcare professionals - and anyone who wants to conduct health research - in the design and development of a health research project.

DOI: https://doi.org/10.48060/tghn.17 


\section{Establish a Research Support Room in your institution / healthcare facility}

Authors: Licia Mota, Cleandro Pires de Albuquerque, Guilherme Dias Malvão, Fernando Araújo Rodrigues de Oliveira, Dayde Lane Mendonça da Silva, Elza Noronha.

Design: Mercedes Rumi, Nicole Feune de Colombi, Steffani Herring-Hall Date of publication: $04-02-2022$

Abstract: Step by step guide to create a Research Support Room, an enduring service to offer support for healthcare professionals - and anyone who wants to conduct health research - in the design and development of a health research project.

DOI: https://doi.org/10.48060/tghn.17

You can browse the online toolkit using the buttons below. Follow it from beginning to end or download the PDF guide to read offline.

\section{\begin{tabular}{|l|l|l|l|}
\hline WHAT WHY HOW - STEP 1 & HOW - STEP 2 & MORE \\
\hline
\end{tabular} DOWNLOAD PDF}
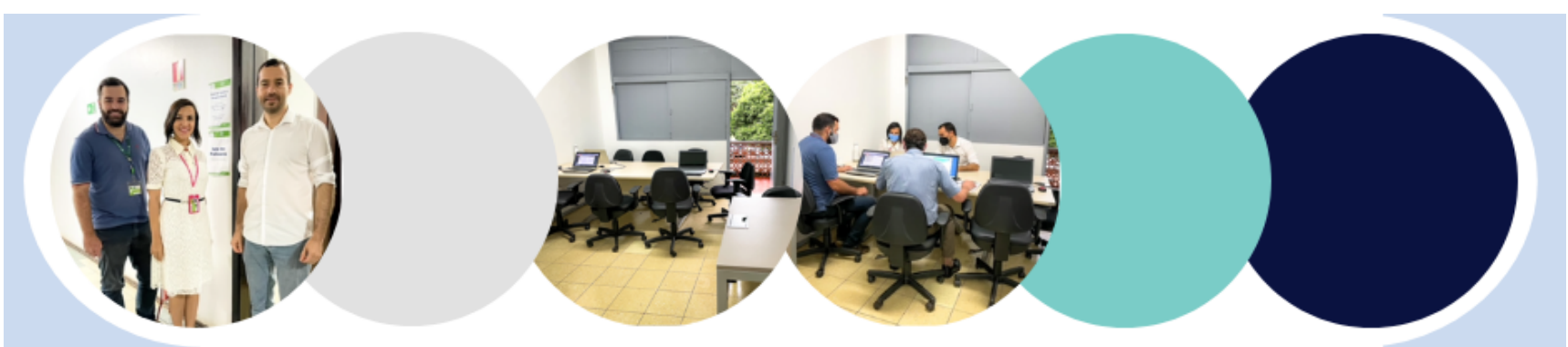


\section{What is a Researcher's Support Room?}

Researcher's Support Room is a dedicated space where research projects are encouraged and nurtured through discussions and guidance (for example, in a healthcare facility).

\section{\begin{tabular}{l|l|l|l|l|} 
HOME & WHY & HOW - STEP 1 & HOW - STEP 2 & MORE
\end{tabular}}

The idea is to offer technical guidance and support to those interested in conducting research. This includes, but is not limited to:

- healthcare professionals (for example; doctors, nurses, lab technicians) wanting to conduct research in their facilities for the first time

- early career researchers looking for mentorship

- experienced scientists seeking for peer-qualified external opinion to improve their investigation

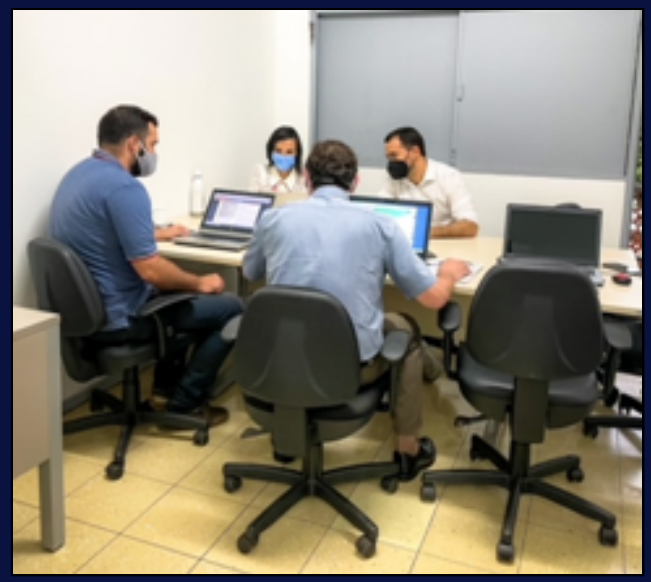

Within this space, discussion regarding research methods and project development take place. The aim is to encourage good quality studies by using the appropriate methods, following ethical standards, and achieving results that are relevant.

\section{Background}

In March 2020, when the COVID-19 pandemic was declared, the University Hospital of Brasilia University of Brasilia assembled a dedicated committee to give institutional support to any relevant COVID-19 research initiatives. Professionals who had long desired to get involved in research, and researchers who had been less active in the field in recent years, were encouraged to join this institutional effort.

"Interacting with researchers, we noticed weakness in the research proposals, open flaws in their methodology, and limited experience with clinical research methods -that could be corrected with little guidance, but also eagerness in joining the global efforts to fight the pandemic and openness to discuss their

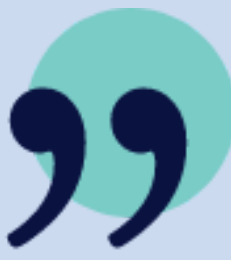
proposals and receive technical and methodological advice." 


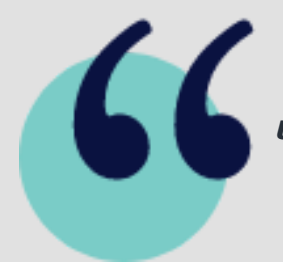

"We realized then that there was an unmet need underlying this scenario that extended beyond the scope of the pandemic represented, for example, by the enthusiasm of professionals in our Institution who had long been desiring to get involved with clinical research, but dealt with methodological and

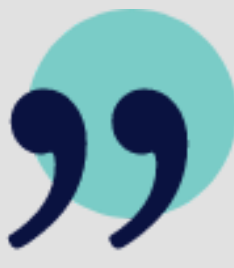
other practical difficulties that kept them apart from achieving the goal."

The University Hospital of Brasilia acknowledged the unmet need as an opportunity and created the Researcher's Support Room in October 2020, an enduring service to offer support for healthcare professionals - and anyone who wants to conduct health research - in the design and development of any health research project.
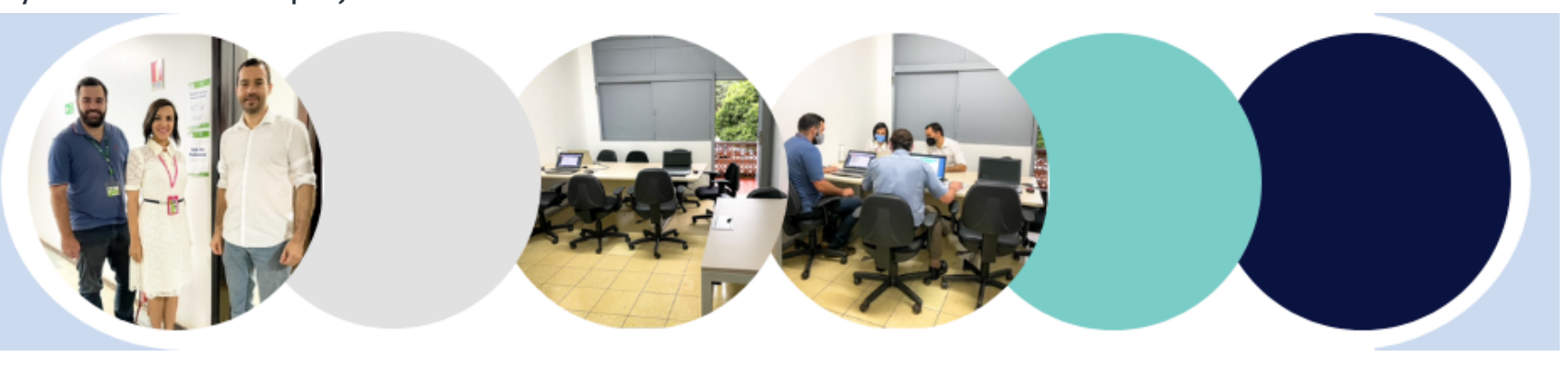

\section{Why create a Researcher's Support Room?}

\begin{tabular}{l|l|l|l|}
\hline HOME WHAT HOW - STEP 1 & HOW - STEP 2 & MORE \\
\hline
\end{tabular}

With proper guidance and methodological support, most professionals who seek aid from the Researcher's Support

Room will increase participation in clinical research, resulting in more evidence and thus improvement of clinical practice.

The Researcher's Support Room is important too for networking and enhancing or catalyzing collaborations and networks between scientists seeking support and other more experienced researchers who work in the specific field that can offer mentoring.

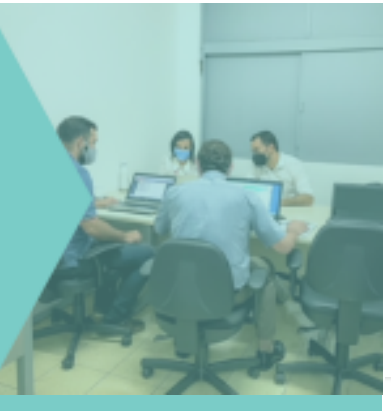


The Researcher's Support Room is important too for networking and enhancing or catalyzing collaborations and networks between scientists seeking support and other more experienced researchers who work in the specific field that can offer mentoring.

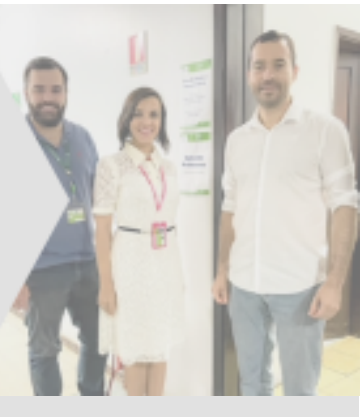

Also, it provides a platform to motivate candidates and further their motivation to seek formal post-graduation programmes, for sponsorship and supervision.

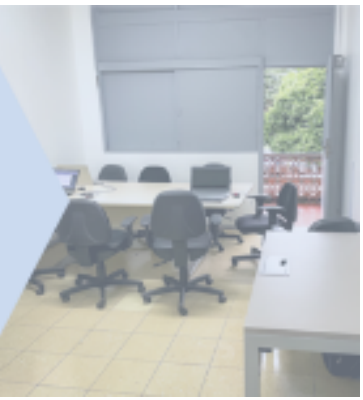

\section{Impact of the Researcher's Support Room in the University Hospital of Brasilia}

Since the Researcher's Support Room started in October 2020, in the first year it has run more than 115 online and in-person technical and methodological advising sessions related to more than 40 projects, and serving more than 80 interested professionals. Further, 16 researchers entered a formal postgraduate (Master's or PhD) degree program under direct assistance from the Room, by offering methodological guidance or through intermediating access to faculty advisors in the University of Brasilia and other Institutions.

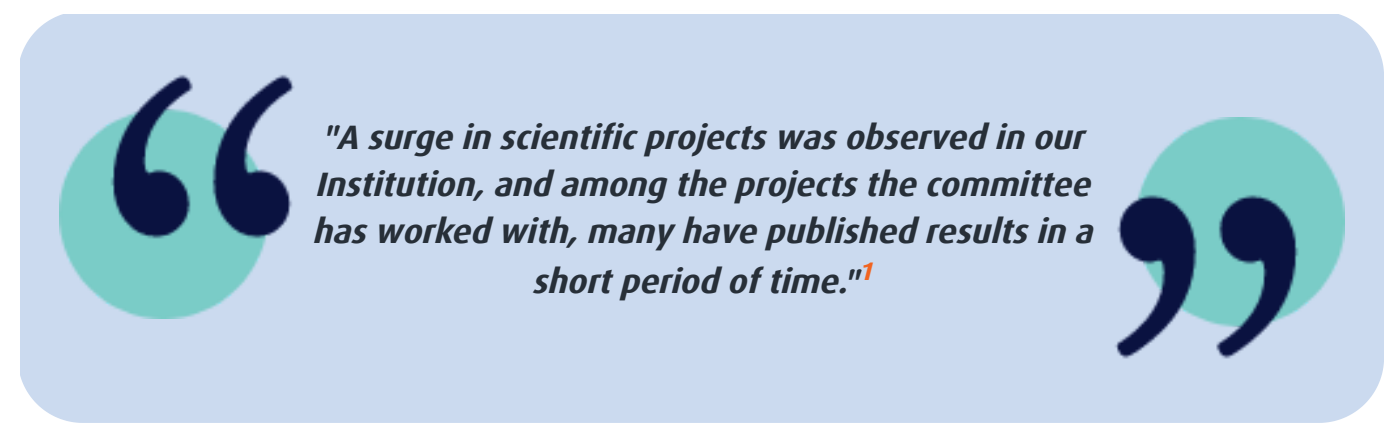

Given its accrued experience in mapping and dealing with researchers' difficulties, the Room has also been invited to participate, along with other institutional counterparts, in the ongoing revision and development of the hospital's overall research policy. Finally, the Room became a bridge between research groups in our hospital and in Faculties in the University of Brasilia and other Institutions, even abroad. 
"The University Hospital of Brasilia despite being a tertiary healthcare facility, focused in highcomplexity assistance, is small in its size and workforce. Thus, these achievements in so little time are surprising even to us. We believe we are contributing to solve unmet needs in our hospital, through the

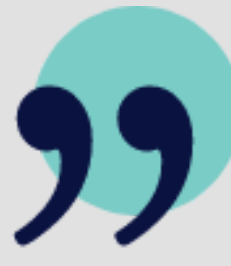
Researcher's Support Room, and that this Project (once replicated) could also benefit other hospitals where clinical researchers might find challenges like those we have been observing in our Institution."

\section{References}

${ }^{1}$ Pinho RDNL, Costa TF, Silva NM, Barros-Areal AF, Salles AM, Oliveira AP, Rassi C, Valero CEB, Gomes CM, Mendonça-Silva D, Oliveira F, Jochims I, Ranulfo I, Neves JBS, Oliveira L, Dantas MN, Rosal M, Soares M, Kurizky P, Peterle VU, Faro YF, Gomides AP, da Mota L, Albuquerque C, Simaan CK, Amado VM. Mental Health and Burnout Syndrome Among Postgraduate Students in Medical and Multidisciplinary Residencies During the COVID-19 Pandemic in Brazil: Protocol for a Prospective Cohort Study. JMIR Res Protoc. 2021 Jan 19;10(1):e24298. doi: 10.2196/24298. PMID: 33290246; PMCID: PMC7817252.

Schulte HL, Brito-Sousa JD, Lacerda MVG, Naves LA, de Gois ET, Fernandes MS, Lima VP, Rassi CHRE, de Siracusa CC, Sasaki LMP, Cerqueira SRPS, de Albuquerque CP, Reis APMG, Gomes CM, Kurizky PS, da Mota LMH, Espindola LS. SARS-CoV-2/DENV co-infection: a series of cases from the Federal District, Midwestern Brazil. BMC Infect Dis. 2021 Jul 31;21(1):727. doi: 10.1186/s12879-021-06456-2. PMID: $34332542 ;$ PMCID: PMC8325531.

Kurizky P, Nóbrega OT, Soares AASM, Aires RB, Albuquerque CP, Nicola AM, Albuquerque P, TeixeiraCarvalho A, Naves LA, Fontes W, Luz IS, Felicori L, Gomides APM, Mendonça-Silva DL, Espindola LS, Martins-Filho OA, de Lima SMB, Mota LMH, Gomes CM. Molecular and Cellular Biomarkers of COVID-19 Prognosis: Protocol for the Prospective Cohort TARGET Study. JMIR Res Protoc. 2021 Mar 4;10(3):e24211. doi: 10.2196/24211. PMID: 33661132; PMCID: PMC7935398.

Fernandes GM, Motta F, Sasaki LMP, Silva ÂPD, Miranda AM, Carvalho A0, Gomides APM, Soares AASM, Santos ACMD Jr, Alves CO, Gomes CM, Siracusa CC, Araújo DA Jr, Mendonça-Silva DL, Jesus JAL, Costa KN, Castro MEC, Kurizky PS, França PS, Tristão R, Pereira YR, Castro LCG, Zaconeta AM, Albuquerque CP, Mota LMHD. Pregnancy Outcomes and Child Development Effects of SARS-CoV-2 Infection (PROUDEST Trial): Protocol for a Multicenter, Prospective Cohort Study. JMIR Res Protoc. 2021 Apr 20;10(4):e26477. doi: 10.2196/26477. PMID: 33793409; PMCID: PMC8059788.

Kurizky PS, Cerqueira SRPS, Cunha DV, Albuquerque CP, Aires RB, Mota LMHD, Gomes CM. The challenge of concomitant infections in the coronavirus disease 2019 pandemic era: Severe acute respiratory syndrome coronavirus 2 infection in a patient with chronic Chagas disease and dimorphic leprosy. Rev Soc Bras Med Trop. 2020 Nov 6;53:e20200504. doi: 10.1590/0037-8682-0504-2020. PMID: 33174962; PMCID: PMC7670735. 


\section{Step 1 - How does the Researcher Support Room Work?}

\section{HOME WHAT WHY HOW - STEP 2 MORE}

\section{Setting up the Research Support Room}

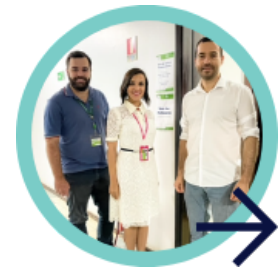

\section{Human Resources}

- Qualified research advisors

- with formal training and experience in clinical research and scientific methodology are required.

- ideally and whenever possible, each project should be assessed and discussed by two simultaneous advisors.

- Having a statistician in the Room is useful, but it is not essential if the advisors have a good understanding of quantitative methods in clinical research (including basic statistics).

- If possible, it is helpful to have an admin staff who organises the Room's agenda.

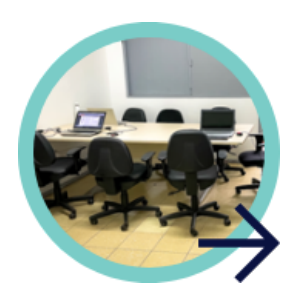

\section{Physical Resources}

- A suitable room inside the hospital or institution. This one can be shared with other groups but not at the same time.

- A large table for the meeting. Sometimes there can be a group of up to 8-10 researchers involved in the same project.

- A computer with access to the internet, as some virtual meeting platforms or software for online appointments, will be required.

\section{Time}

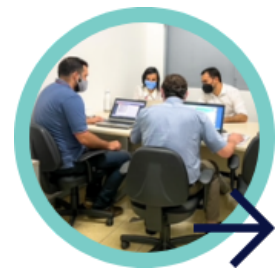

The most important element for the Researcher's Support Room to be successful is time. Carefully reading the projects and understanding the aims, interests and ideas of the researchers is essential to identify and suggest improvements. Research advisors should have time allocated each week for the Research Support Room activities.

- The total amount of time required to run the project will depend on the local demand.

"In the University Hospital of Brasilia, for instance, the Researcher's Support Room is open to the public (upfront reservation required) for two 4-hour periods per week. The secretary is available to receive appointment requests during regular working hours, five days a week." 


\section{Step 2 - How does the Researcher Support Room Work?}

\section{HOME WHAT WHY HOW - STEP 1 MORE}

\section{Day-to-day operations}

Teams or individuals seeking support will first make an appointment.

- Each session lasts 1.5 hrs

- The meetings can be run online or in person, according to the researchers' preferences

\section{First \\ Encounter}

\section{Subsequent \\ Encounters}

\section{The Research Question}

- The team will focus on understanding the real interests of the researcher(s) and help towards developing a good research question.

- Many will have only a general idea about what they want to study at that moment. Others will bring a research project in hand.

- Either way, the team will go through these questions in detail, aiming at helping the researcher to end up with one or more clear, focused, well structured, relevant, ethical, and feasible research questions that should still be interesting and motivating to them.

- It may take one or more encounters to achieve a proper research question.

Once the research question is established (and a testable hypothesis is clearly formulated in case of analytical studies), proceed to review in detail, together with the researcher(s), the methods required to answer the question.

Focus on the general study design, including:

- a clear delimitation of the target population

- sampling techniques and sample size

- recruitment, randomisation (when appropriate)

- interventions, comparisons

- outcomes and their timeframes

- variables in study

- measurement tools

- statistical methods

- data analysis

- ethical requirements 


\section{Final}

Encounters

\section{Implementation}

Finally, address the operational aspects of the project:

- project submission to institutional review boards;

- protocol registering and availability through public repositories;

- technical expertise requirements;

- access to specific resources needed (equipment, tools, materials, etc.);

- standardised procedures to data collection and monitoring;

- research budget and funding sources; and

- realistic timelines for conducting all the research processes.

Going through all thesteps may take several encounters, depending on the previous expertise of the researcher(s). 


\section{Further reading and useful materials}

- Protocol Development Steps toolkit: find guidance on all elements of Protocol development in addition to practical advice such as how to navigate regulations and guidelines, the importance of community engagement, and tips on collaborative writing.

- Study Cycle Main Resources: Useful documents and links divided into sections: Study Design and Planning; Ethics and Regulations; Study Procedures; Data Collection, Management, and Analysis; and Study Operations

- Quality Management Toolkit: set forth the principles for good research practices, helping teams to consider the quality practices and processes of their study, through the actions and measures that are carried out by the team to ensure the quality and consistency of the study results they wish to achieve.

- Study Walk-through: translate your protocol into an accurate and successful study. This toolkit describes the study walkthrough approach, why it might be of benefit to your study and how you can successfully implement the method using practical examples and 'How to' guidance.

\section{- Data Science:}

- Data Management Basics

- Data Management Steps

- Data Management Plan

- Access free online short course: Introduction to Data Management for Clinical Research Studies

- Statistics for beginners: A presentation on basic statistics in clinical research [PDF, 2.7mb]

- An introduction to basic statistics workshop

- Completing a statistical analysis plan: A video on completing a prospective, fixed statistical analysis plan, prior to study initiation
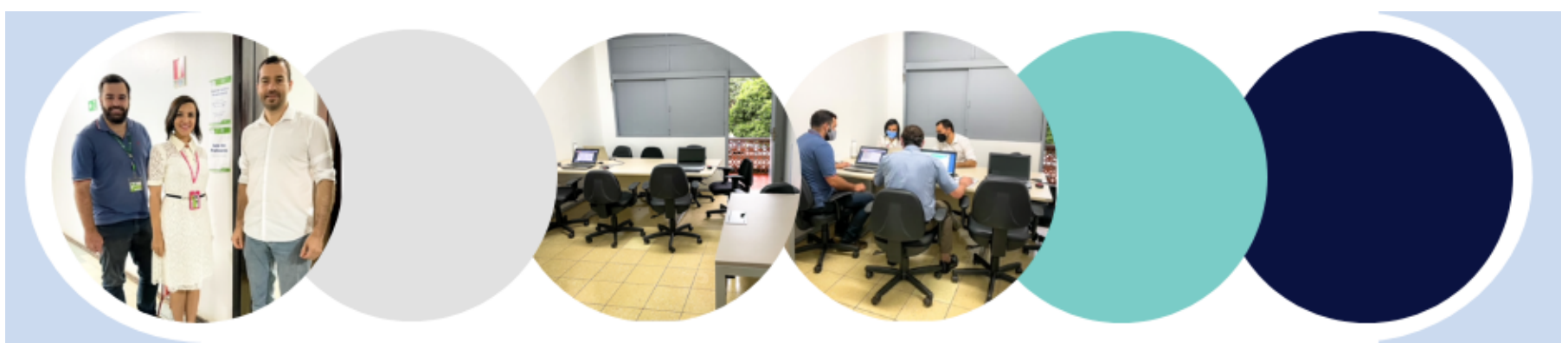